\title{
A Reconfigurable Power-Conscious Core Wrapper and its Application to SOC Test Scheduling
}

\author{
Erik Larsson and Zebo Peng \\ Embedded Systems Laboratory \\ Linköpings Universitet, Sweden
}

\begin{abstract}
$^{1}$
This paper presents a novel reconfigurable powerconscious core test wrapper and discusses its application to optimal power-constrained SOC (system-on-chip) test scheduling. The advantage with the proposed wrapper is that at each core it allows (1) a flexible TAM (test access mechanism) bandwidths, and (2) a possibility to select the appropriate test power consumption. Our scheduling technique, an extension of a preemptive scheduling approach, produces optimal solutions in respect to test time, and selects wrapper configurations in a systematic way that implicitly minimizes the TAM routing and the wrapper logic. Experimental results show the efficiency of our approach.
\end{abstract}

\section{Introduction}

Test power consumption and test time minimization are becoming major challenges when developing test solutions for core-based designs. These problems can be tackled by:

- design for low power testing where the system is designed to minimize test power consumption, which allows consequently testing at a higher clock frequency $[5,21,22,24]$, and

- test scheduling where the tests are organized in such a way that the test time is minimized while considering test power limitations and test conflicts $[1,4,6,7,8,9$, $11,12,13,14,15,16,17,18]$.

A core-based design is composed of pre-defined cores, UDL (user-defined logic) blocks and interconnections. From a testing perspective, all testable units, that is core logic, UDL blocks and interconnections, are defined as cores. The TAM (Test Access Mechanism), the set of wires connecting the ATE (Automatic Test Equipment) and the cores under test, is responsible for the test data transportation. A wrapper is the interface between the TAM and a core and it can be in one of the following modes at a time: normal operation, internal test or external test. A core can be either wrapped or unwrapped, which means we have two types of tests: wrapped core test at wrapped cores and cross-core test (interconnection test) at unwrapped cores [19].

1. The research is partially supported by the Swedish National Program on Socware.
We have previously [17]:

- shown that the problem of scheduling wrapped core tests on the TAM is equivalent to independent job scheduling on identical machines,

- made use of a preemptive approach to produce a solution with optimal test time in linear time [2] using reconfigurable wrappers [15], and

- extended the scheduling algorithm to handle wrapped core tests and cross-core tests while preserving the production of an optimal solution in linear time.

The main advantages of our previous approach are that: (1) optimal test time is achieved in linear time, (2) the TAM routing is minimized by assigning a minimum of TAM wires per core, and (3) the reconfigurable wrappers are selected and inserted in a systematic manner to minimize the number of configurations, which minimizes the added logic.

In this paper, we propose a reconfigurable powerconscious (RPC) core test wrapper to regulate the test power at core-level, and we describe its application to optimal test scheduling. The main contributions are:

- the development of a RPC core test wrapper which combines the gated sub-chain scheme presented by Saxena et al. [24] and the reconfigurable core test wrapper introduced by Koranne [15],

- the integration of the RPC wrapper in the preemptive test scheduling approach, and

- the formulation of a power condition that, if satisfied, guarantees that our preemptive test scheduling scheme produces optimal test time.

The advantages of the proposed approach are that we can:

- regulate the power consumption at each individual core, which allows the test clock speed to increase,

- regulate the test power consumption at system level, which should be kept within a given value in order to reduce the risk of over-heating which might damage the system under test,

- select the best TAM size, and

- perform minimization of the TAM routing and the overhead when using the RPC wrapper.

The rest of the paper is organized as follows. Related work is reviewed in Section 2 and our reconfigurable powerconscious test wrapper is introduced in Section 3. In Section 
4 we present our optimal preemptive test scheduling technique, based on a known preemptive scheduling algorithm [2]. In the experiments we have made a comparison with previous approaches and we illustrate the advantages with our wrapper and its use in the proposed scheduling approach in Section 5. The paper is concluded with conclusions in Section 6.

\section{Background and Related Work}

Figure 1 shows an example of a core-based system consisting of five cores, a TAM and an externally placed ATE (serving as test source and test sink). The test source, where the test stimulus are stored, and the test sink, where the test responses are saved, are connected to the $N_{\text {tam }}$ wire wide TAM. The test source feed test stimulus to the cores via the TAM and the produced test responses from the cores are also transported via the TAM to the test sink. The cores are equipped with a test method; core 1 is, for instance, scan tested. A wrapper is the interface between a core and the TAM. Its main task is to connect the scan chains and the wrapper cells at a core into a set of wrapper chains, which are connected to the TAM. Cores with a dedicated wrapper, such as core 1,2, and 3, are wrapped while cores without a dedicated wrapper, such core 4 and 5 , are unwrapped. A core test is a test at a wrapped core, while a cross-core test is a test at an unwrapped core. The execution of a core test and a cross-core test therefore differs from each other. A core test is performed by placing its wrapper cells in internal test mode, and the test stimulus are transported direct from the test source via the TAM to the core. The test responses are transported from the core via the TAM to the test sink. The testing of an unwrapped core (such as core 4 in Figure 1), cross-core test, requires that the wrapper at core 1 and the wrapper at core 2 are both placed in external test mode. Test stimulus are then transported from the test source on the TAM via the wrapper cells at core 1 to core 4 . The test responses are transported from core 4 via the wrapper cells at core 2 and the TAM to the test sink.

In the particular example given in Figure 1, the testing of core 1 and core 4 cannot be performed concurrently due to that in both cases the wrapper at core 1 is needed, and a wrapper can only be in one mode at a time. In general, test conflicts such as this one must be considered in the scheduling process. There are mainly two types of test

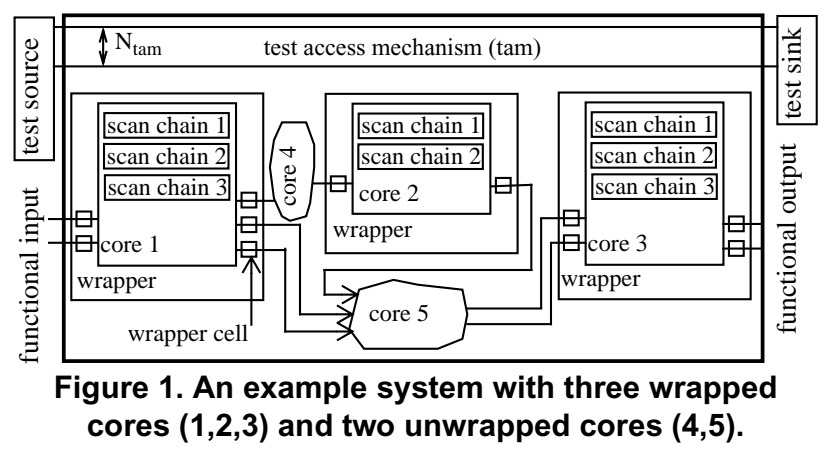

conflicts: (1) TAM wire conflicts and (2) core wrapper conflicts, respectively.

Several test scheduling techniques have been proposed $[1,4,6,7,8,9,11,12,13,14,15,16,17,18]$. Chou et al. proposed a technique for general systems where each test has a fixed test time and a fixed power consumption value [4]. The objective is to organize the tests in such a way that the total test application time is minimized while test conflicts and test power limitation are taken in to account [4]. The testing time for a test can often be modified. In scan testing, for example, the test time at a core can be modified by adjusting the number of wrapper chains that the scanned elements are configured into. A low number of wrapper chains at a core reduces the number of occupied TAM wires at the expense of higher testing time. And vice versa. Several wrapper chain configuration and TAM wire assignment algorithms to minimize the test time for core tests at wrapped cores have been proposed $[6,7,8,9,11,12$, 13, 14, 15, 16]. For instance, Iyengar et al. [12] used an ILP (Integer-Linear Programming) approach. Koranne [15] introduced a reconfigurable wrapper with the advantage of allowing $N_{\text {tam }}$ wrapper chain configurations per wrapped core. In order to minimize the added overhead due to the flexible wrapper, a limited number of cores are selected to have a reconfigurable wrapper prior to the scheduling. Iyengar et al. [11] and Huang et al. [9] proposed scheduling techniques for core tests under power constraints with fixed test power consumption for each test. Nicolai and AlHashimi [21] proposed a technique to minimize useless switches, and Gerstendörfer and Wunderlich [5] introduced a technique to disconnect the scan-chains from the combinational logic during the shift process, both leading to a lower power consumption during test, which reduces test time by allowing clocking at a higher frequency. For the same purpose, Saxena et al. [24] proposed an approach to gate sub-chains.

\section{A Novel Reconfigurable Power-Conscious Core Test Wrapper}

The RPC (reconfigurable power-conscious) test wrapper we propose combines the gated sub-chain approach proposed by Saxena et al. [24] and the reconfigurable wrapper introduced by Koranne [15]. The basic idea in the approach proposed by Saxena et al. [24] is to use a gating scheme to lower the test power dissipation during the shift process. Given a set of scan-chains as in Figure 2 where the three scan-chains are connected into a single chain. During the shift process, all scan flip-flops are active, leading to high switch activity in the system and therefore high power consumption. However, if a gated sub-chain scheme is introduced (Figure 3), only one of the three chains is active at a time during the shift process. The advantage is that the switch activity is reduced in the scan-chains and also in the clock tree distribution while the test time remains the same in the two cases [24]. 


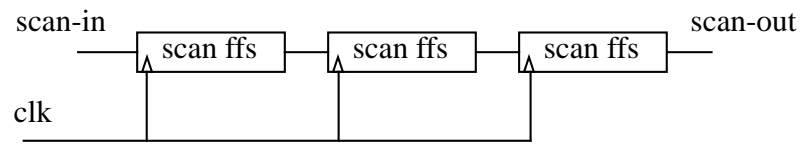

Figure 2. Original scan chain [24].

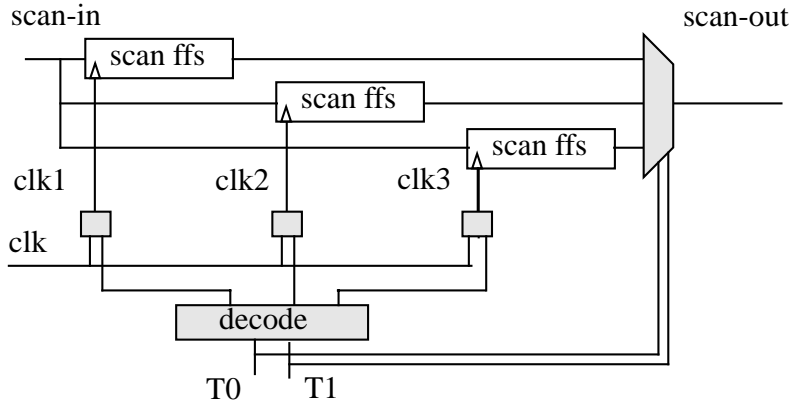

Figure 3. Scan chain with gated sub-chains[24].

The wrapper proposed by Koranne allows, in contrast to approaches such as Boundary Scan, TestShell and P1500, several wrapper chain configurations [15]. The main advantage is the increased flexibility in the scheduling process. We use a core with 3 scan chains of length $\{10,5$, $4\}$ to illustrate the approach. The scan-chains and their partitioning into wrapper chains are specified in Table 1.

\begin{tabular}{ccc}
\hline TAM width & Wrapper chain partitions & Max length \\
\hline 1 & {$[10,5,4]$} & 19 \\
2 & {$[(10),(5,4)]$} & 10 \\
3 & {$[(10),(5),(4)]$} & 10 \\
\hline
\end{tabular}

Table 1. Scan chain partitions.

For each TAM widths $(1,2$, and 3$)$ a di-graph (directed graph) is generated where a node denotes a scan-chain and the input TAM, node I (Figure 4). An arc is added between two nodes (scan-chains) to indicate that the two scan-chains are connected, and the shaded nodes are to be connected to the output TAM. A combined di-graph is generated as the union of the di-graphs. Figure 5 shows the result of the generated combined di-graph from the three di-graphs in Figure 4. The indegree at each node (scan-chain) in the combined di-graph gives the number of signals to multiplex. For instance, the scan chain of length 5 has two
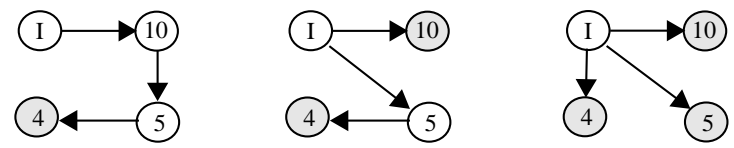

(a) 1 wrapper-chain (b) 2 wrapper-chains (c) 3 wrapper-chains

Figure 4. Di-graph representations.

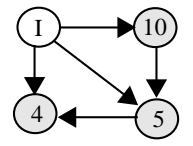

Figure 5. The union of di-graphs in Figure 4.

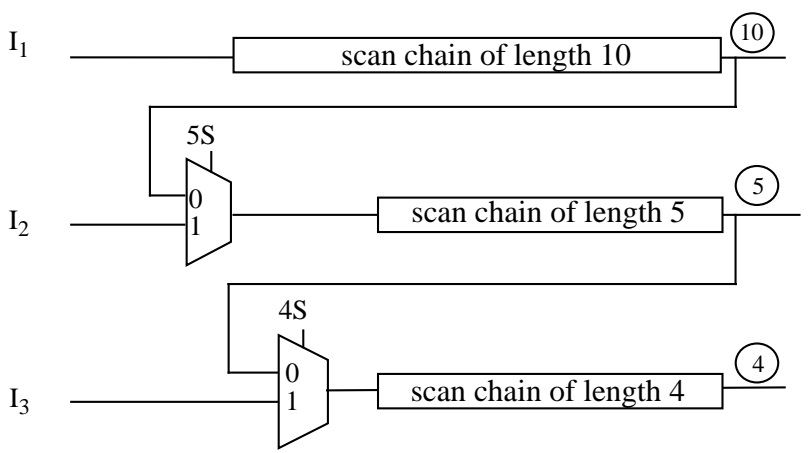

Figure 6. Multiplexing strategy [15].

input arcs, which in this example means that a multiplexer selecting between an input signal and the output of the scan chain of length 10 is needed. The multiplexing for the example is outlined in Figure 6.

Our combined approach works in two steps. First, we generate the flexible wrapper using Koranne's approach. Second, we add clock gating, which means we connect the inputs of each scan-chain to the multiplexers, which is to be compared to connecting the outputs of each scan-chain as in the approach by Koranne. We illustrate our approach using the scan chains specified in Table 1. The result is given in Figure 7, and the generated control signals are in Table 2.

The advantages with our approach are that we gain control of the test power consumption at each core, and we do not require the extra routing needed with Koranne's approach, as illustrated in Figure 8.

We could make use of the RPC wrapper at all cores, which would lead to a high flexibility since we could reconfigure the wrapper into any configuration. However, in

\begin{tabular}{ccccc}
\hline $\begin{array}{c}\text { Wrapper } \\
\text { chains }\end{array}$ & T0 T1 T2 & 5S 4S & S1 S2 & clk10 clk5 clk4 \\
\hline 3 & 000 & 11 & 00 & 111 \\
\hline 2 & 001 & $1 \times$ & 00 & 100 \\
\hline 1 & 010 & 10 & 01 & 011 \\
\hline & 1011 & $\mathrm{xx}$ & $0 \mathrm{x}$ & 100 \\
& 101 & $0 \mathrm{x}$ & 10 & 010 \\
\hline
\end{tabular}

Table 2. Control signals.

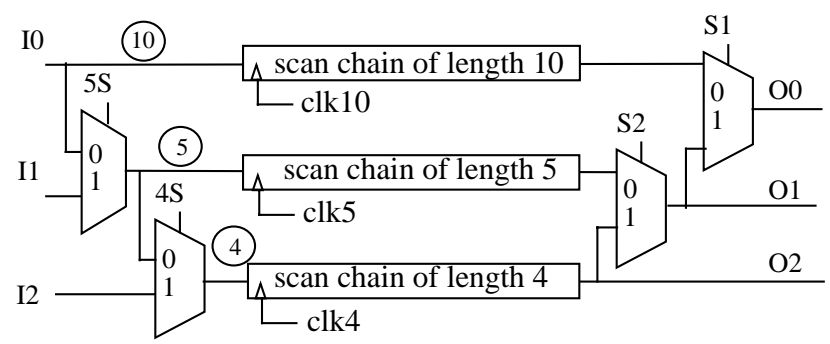

Figure 7. Our multiplexing and clocking strategy. 


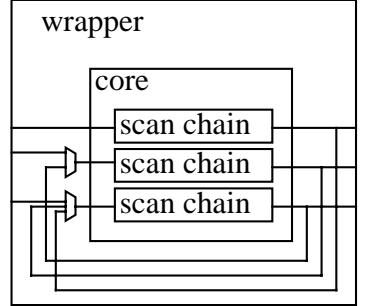

(a) Koranne's routing [15].

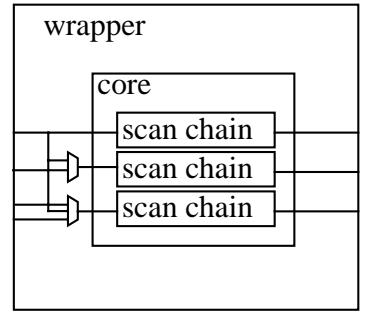

(b) Our approach.

Figure 8. Wrapper routing.

order to minimize the overhead, we will use a systematic approach to select cores and number of configurations at each core (described below).

\section{Optimal Power-constrained Test Scheduling}

In this section we describe our power-constrained test scheduling technique that produces optimal test time in linear time while scheduling both core tests and cross-core tests. The approach also selects the wrapper configurations.

\subsection{Optimal Scheduling of Core Tests}

The test scheduling problem of core tests is equal to the independent job scheduling on identical machines since each test $t_{i}$ at a core $c_{i},(i=1,2, \ldots, n)$ with testing time $\tau_{i}$ is independent on all other core tests, and each TAM wire $w_{j}$ $\left(j=1,2, \ldots, N_{\text {tam }}\right)$ is an independent machine used to transport test data [17]. The LB (lower bound) of the test time for a given TAM width $N_{\text {tam }}$ can be computed by [2]:

$$
L B=\max \left\{\max \left(\tau_{i}\right), \sum_{i=1}^{n} \tau_{i} / N_{\text {tam }}\right\}
$$

The problem of independent job scheduling on identical machines can be solved in linear time $(O(n)$ for $n$ tests) by using preemption [2]: assign tests to the TAM wires successively, assign the tests in any order and preempt tests into two parts whenever the LB is reached. Assign the second part of the preempted test on the next TAM wire starting from time point zero.

An example (Figure 9) illustrates the approach where the five cores and their test times are given. The LB is computed to 7 (Equation 1) and due to that $\tau_{i} \leq \mathrm{LB}$ for all tests; the two parts of any preempted test will not overlap. The scheduling proceeds as follows: The tests are considered one by one, for instance, starting with a test at $c_{1}$ scheduled at time point 0 on wire $w_{1}$. At time point 4 , when the test at $c_{1}$ is finished, the test at $c_{2}$ is scheduled to start. At time point 7 when LB

$$
\begin{aligned}
& N_{\text {tam }}=3 \\
& c_{i} \mid 12345 \\
& \hline \tau_{i} \mid 45345
\end{aligned}
$$

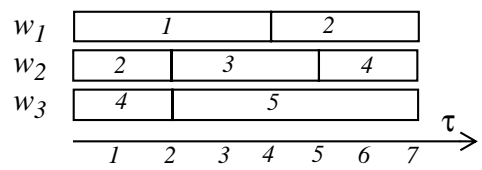

Figure 9. Optimal TAM assignment and preemptive scheduling. is reached, the test at $c_{2}$ is preempted and the rest of the test is scheduled to start at time 0 on wire $w_{2}$. Therefore the test at $c_{2}$ is partitioned into two parts. In execution of the test at $c_{2}$, the test starts at wire $w_{2}$ at time point zero. At time point 2 , the test is preempted and resumed at time point 4 . The test ends at time point 7 . At the preemption of a test, another wire is assigned to the core and a multiplexer is added for wire selection. For the test of $c_{2}$, a multiplexer is added to select between $w_{1}$ and $w_{2}$.

In general preemptive scheduling, extra time is introduced at each preemption point due to the need to set up a job and also to save its state. In our case, the machines are the wires and no extra time is needed to set up and save the state. Also, in testing no other tasks are performed at the cores but testing, i.e. the core's state can be left as it is until the testing continues. The advantage is that the state of the core is already set and testing of it can start at once.

Assume that a core has a wrapper-chain of length $l$ ( $l$ cycles are needed to perform a shift-in of a new vector and a shift-out of the previous test response). If the test is preempted when $x \%$ of the $l$ cycles are shifted in it means that when the test restarts $x \%$ of the new test vector is already loaded and $x \%$ less cycles are needed in the first shift process, i.e. there is no time overhead due to setting up and saving the state of a core; all tests can be stopped at LB.

Finally, in some cases, such as for some types of memories such as DRAMs, the testing cannot be preempted. For instance, assume that test $t_{2}$ cannot be preempted as in Figure 9. In such a case, when LB is met, the scheduling algorithm restarts at LB (and not at time 0) and moves towards zero. The resulting schedule is in Figure 10. Note that, test $t_{2}$ now makes use of one wire during time point 4 to 5 and two wires during time 5 to 7 , which is possible using the reconfigurable wrapper. This overlapping is further discussed below.

$$
\begin{aligned}
& N_{\text {tam }}=3 \\
& c_{i} \mid 12345 \\
& \hline \tau_{i} \mid 45345
\end{aligned}
$$

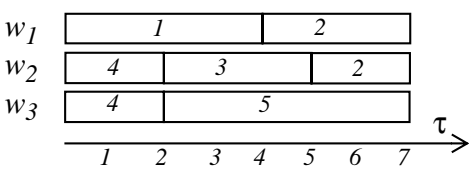

Figure 10. Optimal TAM assignment and preemptive scheduling where test $t_{2}$ cannot be interrupted.

\subsection{Transformations for Optimal TAM Utilization}

A long test time for one of the test in the system may limit the solution, i.e. LB is given by the test time of a test $\left(\max \left(\tau_{i}\right)\right.$ in Equation 1$)$. In such a case, the test time can be reduced by assigning more TAM wires so that the length of the wrapper chains becomes shorter. Our approach is straight forward, we remove $\max \left(\tau_{i}\right)$ from Equation 1:

$$
L B=\max \sum_{i=1}^{n} \tau_{i} / N_{t a m} .
$$


When LB is computed, we use the scheduling approach illustrated above (Figure 9). For illustration, we use the same example but with a wider TAM $\left(N_{t a m}=7\right)$. The scheduling result is presented in Figure 11. A test may now overlap in using the wires (machines). For instance, the test at $c_{1}$ uses wire $w_{1}$ and $w_{2}$ during time period 0 to 1 and only wire $w_{1}$ during period 1 to 3 . A reconfigurable wrapper is required to solve this problem.

We solve the overlapping problem in two consecutive steps: partitioning of the tests, and inserting of reconfigurable wrappers. After assigning TAM wires to all tests, we determine the partitions, which is illustrated in Figure 11. For instance, in partition 1 of the test at $c_{2}, w_{3}$ is used during period $\tau_{21}$ and in partition 2 of the test at $c_{2}, w_{2}$ and $w_{3}$ are used during period $\tau_{22}$. From this we can determine that two wrapper chains are initially needed and then a single wrapper chain is needed. In total, two configurations are needed for core $c_{2}$. The generic partitioning of a test's usage of wires over the testing time is given in Figure 12. For each test, a start time start $_{i}$ and an end end $_{i}$ are assigned by the algorithm, respectively. The number of partitions, which will be the number of configurations, is computed for each test by the algorithm given in Figure 13. If the test time $\tau_{i}$ for a test $t_{i}$ is below LB, only one configuration is needed. A multiplexer might be required for wire selection if start $_{i}>$ end $_{i}$. From the algorithm, we find that the maximal number of partitions per test is three, which means we in the worst case have to use three configurations per core. The wrapper logic is then

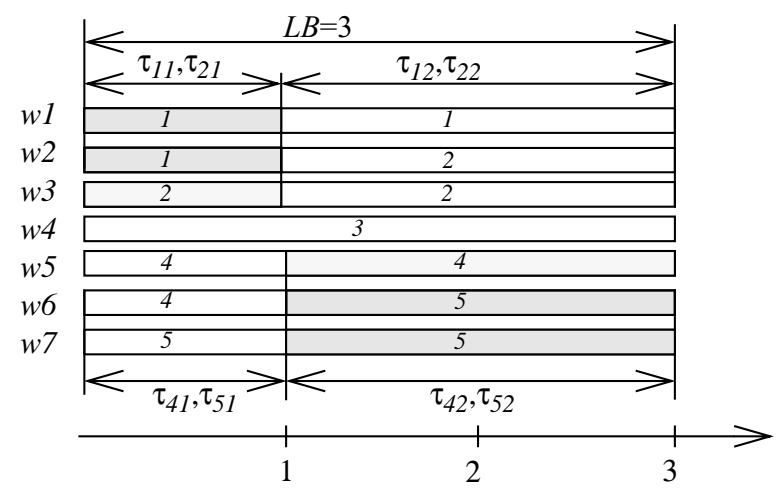

Figure 11. Partitioning of the schedule in Figure 11.

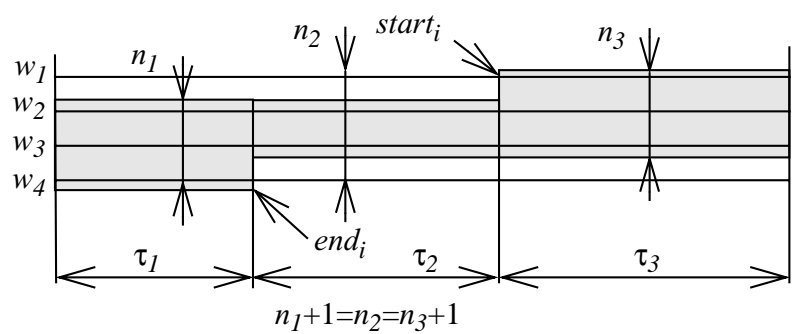

Figure 12. Bandwidth requirement for a general test.

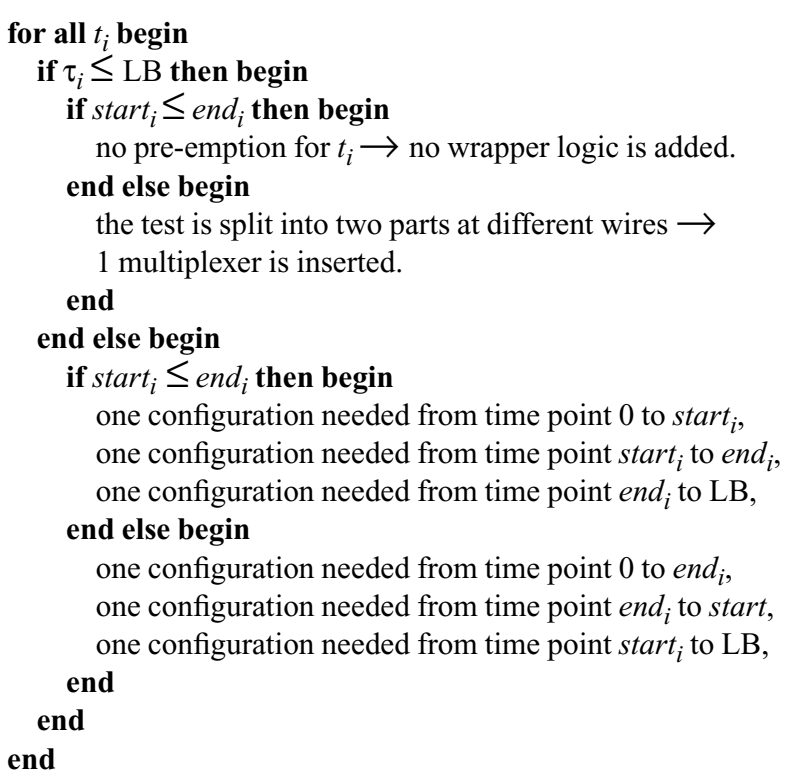

Figure 13. Algorithm to determine wrapper logic.

in range $|\mathrm{C}| \times 3 \times$ technology parameter (maximum 3 configurations per core). In the approach by Koranne [15] the added logic, if a reconfigurable wrapper is added at all cores, is given by $|\mathrm{C}| \times N_{\text {tam }} \times$ technology parameter.

\subsection{Cross-Core Test Scheduling}

There are no wrapper conflicts among core tests since each core has its dedicated interface to the TAM. For cross-core tests, on the other hand, there is not a dedicated interface to the TAM and wrapper conflicts must therefore be taken into account.

Test conflicts can be modelled using a resource graph [4]. The system in Figure 1 is modelled as a resource graph as in Figure 14, where the nodes represents the tests and the resources. A resource may consist of cores and wrapper cells, which are explicitly captured as shown in Figure 14. An edge between a test and a resource (core or a wrapper cell) indicates that the test requires the resource during testing. The test conflicts are due to that the wrapper cells can only be in one mode at a time: In core testing the wrapper cells are in internal test mode while in cross-core test they are in external test mode. In Figure 14, we denote arcs from core tests with $(i)$ - internal mode and arcs from

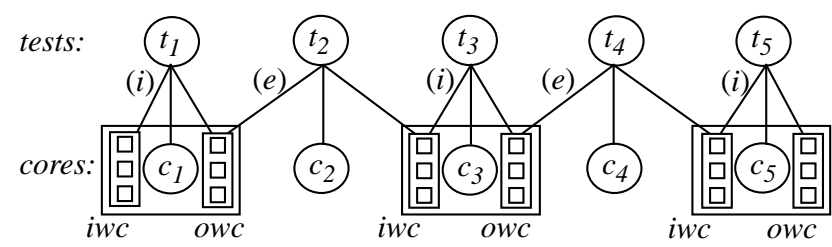

iwc-input wrapper cells wrapper mode: $i$-internal mode owc-output wrapper cells $\quad e$-external mode

Figure 14. A resource graph of the system in Figure 1 
cross-core tests with $(e)$ - external mode. Breaking the graph into two resource graphs, one for core tests and one for cross-core tests makes it possible to schedule all core tests with the algorithm in section 4.1.

For a cross-core test, test vectors are transported from the TAM to a wrapped core placed in external test mode, which is feeding the test vectors to the unwrapped core (the target for the cross-core test). The test responses are captured at a wrapped core also in external test mode and then the test responses are fed to the TAM. A cross-core test involves therefore three cores. In Figure 1 a cross-core test at $c_{4}$ is performed by setting the wrappers at $c_{1}$ and $c_{2}$ in external test mode, and then test vectors are transported to $c_{4}$ through the wrapper at $c_{1}$ and the test response from $c_{4}$ is transported to the TAM using the wrapper at $c_{2}$. This demonstrates a cross-core test with a one-to-one mapping where the wrapper cells at the functional outputs at $c_{1}$ are connected via $c_{4}$ to the functional input wrapper cells at $c_{2}$.

Several other mapping combinations are possible for the wrapper input and wrapper output cells, including one-tomany, many-to-one and many-to-many. These mappings cover all combinations and we assume that each functional input and output can be in only one such mapping and in only one test set. In Figure 1, for instance, a functional output wrapper cell at $c_{1}$ cannot be in one test set with an input wrapper cell at $c_{5}$ and in another test set with an input cell at $c_{3}$. However, a wrapper cell at $c_{1}$ can be in the same test set as a wrapper cell at $c_{3}$ and at $c_{5}$. In some cases, the functional inputs and outputs at a wrapped core may be connected to different cores. Figure 1 shows such an example where the outputs at $c_{1}$ are partitioned into two sets, one set used by $c_{2}$ and $c_{4}$ and another set used by $c_{3}$ and $c_{5}$. However, these partitions operates independently when the wrapper is in external test mode. Therefore there is no conflict.

We have above shown that partitioning the tests into two distinct test phases, core tests and cross-core tests, eliminates the wrapper conflicts between the set of core tests and the set of cross-core tests. We make use of this property and divide the test scheduling into two separate parts, core testing followed by cross-core testing. The partition of the tests means we divide the tests into a core test part given by $L B_{c t}$ and a cross-core test part given by $L B_{i c t}$. To illustrate, we take the example in Figure 9 assuming that the test at $c_{2}$ and $c_{4}$ are cross-core tests, which means that executing the test at $c_{2}$ entails concurrent testing at $c_{1}$ and at $c_{3}$, and testing $t_{4}$ entails concurrent testing at $c_{3}$ and at $c_{5}$. The core tests are performed at core $c_{1}, c_{3}$ and $c_{5}$ and the cross-core tests are at core $c_{2}$ and $c_{4}$. The lower bound for the core tests: $L B_{c t}=(4+3+5) / 3=4$ and the lower bound for the cross-core tests: $L B_{i c t}=(5+4) / 3=3$, i.e. $L B=L B_{c t}+L B_{i c t}$. One test schedule is presented in Figure 15.

The test scheduling algorithm consists of four steps:
1. Compute $L B_{c t}$ (lower bound) for the core tests,

2. Schedule all core tests,

3. Compute $L B_{i c t}$ for the cross-core tests, and

4. Schedule all cross-core tests.

The algorithm starts by selecting a core and assigning it to wire zero at time zero. If the core's test time is higher than $\mathrm{LB}$, a new wire is used. The test time is reduced until it reaches zero and each time LB is reached, a new wire is added to the test. The start time and the end time of the tests are used when creating the partitions. We observe that the LB defines the test application time and also that all TAM wires are fully utilized, all tests ends at the same time (Figure 11). It means that partitioning the tests into two partitions (core tests and cross-core tests) will still produce an optimal solution.

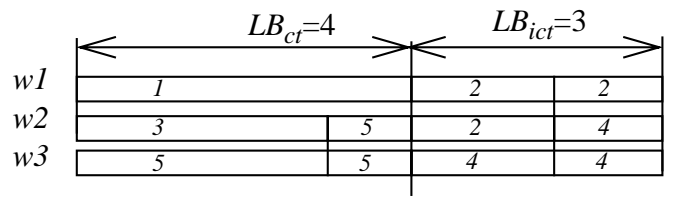

Figure 15. Partitioning of the schedule in Figure 11.

\subsection{Optimal Power-Constrained Scheduling}

Chou et al. introduced a power model where each test is denoted with a fixed power consumption value [4]. Recently a more elaborate model was presented by Rosinger et al. [23]. The power consumption depends on the switching activity in the circuit, and by reducing the switching activity, the power consumption is reduced. Saxena et al. [24] showed by experiments that sub-gating a single scan-chain into three sub-chains reduces the test power to approximately a third. Rosinger et al. proposed a technique to reduce both shift and capture power consumption. The experimental results indicate, in most cases, that the power consumption is lower than the intuitive approximation of dividing the consumption at a single chain with the number of partitions [22]. In this paper, we use a power model based on the results by Saxena et al, which means the power depends on the number and the length of the wrapper chain partitions. However, a more elaborate power model can easily be adopted in our approach.

We use an example to illustrate the test power modelling at scan-chain level (Figure 16). In Figure 16 (a) a single wire is assigned to the core where the three scan chains form a single wrapper chain. The result is that the wire usage is minimized but both the test time and the test power are relatively high. In Figure 16 (b) three TAM wires (one per wrapper chain) are used resulting in a lower test time while the test power consumption remain the same as in Figure 16(a). However, in Figure 16(c), our approach, the same test time is achieved as in Figure 16(a) but at a lower test power consumption. The reduction in test power in this 

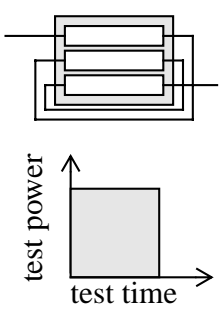

(a)
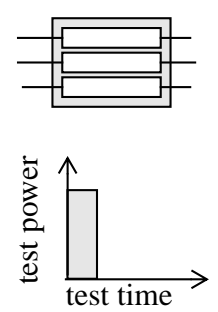

(b)
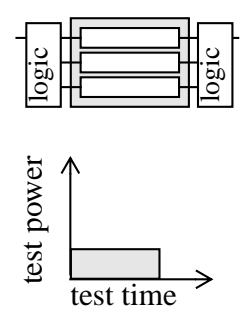

(c)
Figure 16. Core design alternatives.

example is due to that each scan-chain is loaded in a sequence, and not more than one scan-chain is activated at a time.

Our test scheduling technique [17] minimizes the number of TAM wires at each core by assigning as few wires as possible to each core. It means that each wrapper chain includes a high number of scanned elements. This is an advantage since it maximizes the possibility to gate scanchains at each wrapper chain and hence control the test power consumption at the cores.

We assume that the test power at a core is evenly distributed over the scanned elements. The algorithm to compute the power limit $\left(P_{\text {limit }}\right)$ for a system is in Figure 17. At step 2, the LB is computed, and at step 3, the maximal number of required TAM wires are computed. At step 4, the amount of test power consumed by each scan chain, and wrapper cell is computed. At steps 5 and 6, the $N_{\text {tam }}$ values with highest test power are summarized which is the $P_{\text {limit }}$. If $P_{\text {limit }}$ is below $P_{\max }\left(P_{\text {limit }} \leq P_{\text {max }}\right)$, optimal test time can be achieved.

1. Given: a system with $i$ cores, where each core $i$ consists of $f f_{i}$ scanned flip-flops and wrapper cells partitioned into $j$ partitions each of length $s c_{i j}$ (including wrapper cells). The test time $\tau_{i}$ is computed as if all $f f_{i}$ elements are connected to a single wrapper chain. The test power when all $f f_{i}$ elements are active is given by $p_{i}$. $N_{\text {tam }}$ is the TAM bandwidth.

2. Compute $L B$ (lower bound) (algorithm in Section 4.3)

3 . For each core $i$ compute $w_{i}$ as the maximal number of TAM wires that can be assigned to it assuming preemptive scheduling:

$$
w_{i}=\left\lceil\frac{\tau_{i}}{L B}\right\rceil
$$

4. For each scan-chain partition $s_{i j}$ compute its power:

$$
p_{i j}=\frac{p_{i}}{f f_{i}} \times s c_{i j}
$$

5. For all cores: select the $w_{i}$ scan elements with highest power value and sort them descending in a list $L$.

6. For all scan elements in L select the $N_{\text {tam }}$ first and the $P_{\text {limit }}$ is equal to the summation of the $N_{\text {tam }}$ values.

Figure 17. Algorithm to compute the power limit.
We have now a relationship between the TAM bandwidth and the test power. The advantage is that we can use it when we determine the TAM bandwidth; $N_{\text {tam }}$ can be increased as long as $P_{\text {limit }} \leq P_{\text {max }}$. It is also possible to increase the frequency of the test clock in order to minimize the test time as long as $P_{\text {limit }} \leq P_{\text {max }}$.

\subsection{Minimization of TAM Wiring}

The test scheduling approach above minimizes the number of TAM wires assigned to each core. The advantage is that, even if the floor-plan is unknown, the TAM routing cost is minimized since as few TAM wires as possible are assigned to each core. If the floor-plan is known, we can further minimize the TAM routing since the scheduling approach does not require any particular sorting of the tests. We take the system in Figure 9 with $N_{\text {tam }}=7$ resulting in a test schedule as in Figure 11 where the cores are sorted (and numbered) clock-wise as in Figure 18. The advantage is that neighbouring cores share TAM wires. For instance core 2 , which makes use of TAM wire $w_{2}$ as soon as core 1 finish its use of $w_{2}$. Cores placed far away from each other are not sharing TAM wires, such as core 5 and core 3 .

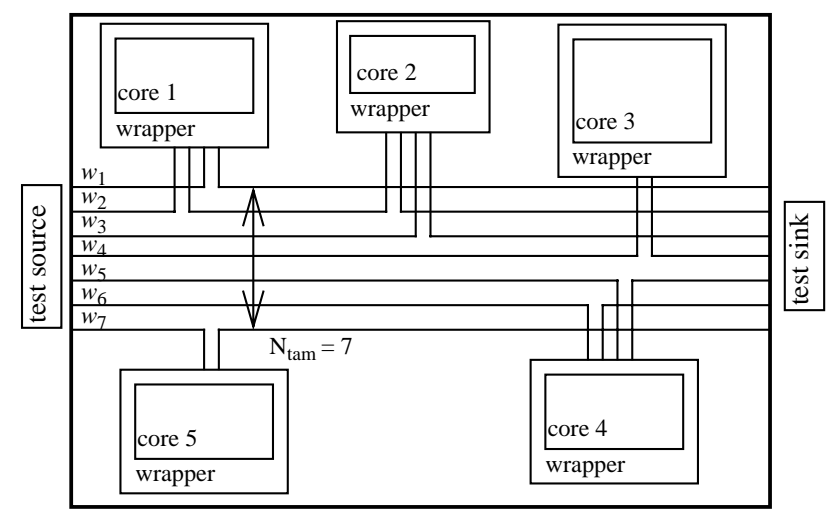

Figure 18. The example system assuming the five wrapped cores to be floor-planned.

\section{Experimental Results}

We have above shown that the test scheduling problem can be solved in linear time by using our proposed RPC wrapper. For illustration, we have made experiments using the P93791 design, one of the largest ITC'02 benchmarks [20]. We have made a test time comparison between our approach and techniques proposed by Goel and Marinissen [6, 7, 8], Huang et al. [9], Iyengar et al. [12, 13, 14], Koranne [15], and Koranne and Iyengar [16]. In our implementation we made use of the wrapper chain algorithm proposed by Iyengar et al. [12]. Similar to all previous approaches, we assume that all tests are core tests. The results are collected in Table 3. In some cases the previously reported results are below the lower bound computed by Goel and Marinissen [6]. These results are 


\begin{tabular}{|c|c|c|c|c|c|c|c|}
\hline \multirow{2}{*}{ Approach } & \multicolumn{7}{|c|}{ Test application time: $T$} \\
\hline & TAM=16 & TAM=24 & TAM=32 & TAM=40 & $\mathrm{TAM}=48$ & TAM=56 & TAM $=64$ \\
\hline Lower bound [6] & 1746657 & 1164442 & 873334 & 698670 & 582227 & 499053 & 436673 \\
\hline Enumerate [12] & 1883150 & 1288380 & 944881 & 929848 & 835526 & 537891 & 551111 \\
\hline ILP [12] & 1771720 & 1187990 & 887751 & $(698583)$ & 599373 & 514688 & 460328 \\
\hline Par eval [13] & 1786200 & 1209420 & 894342 & 741965 & 599373 & 514688 & 473997 \\
\hline GRP[14] & 1932331 & 131084 & 988039 & 794027 & 669196 & 568436 & 517958 \\
\hline Cluster [8] & - & - & 947111 & 816972 & 677707 & 542445 & 467680 \\
\hline TRA [7] & 1809815 & 1212009 & 927734 & 738352 & 607366 & 529405 & 461715 \\
\hline Binpack[10] & 1791860 & 1200157 & 900798 & 719880 & 607955 & 521168 & 459233 \\
\hline CPLEX[15] & 1818466 & $(1164023)$ & 919354 & 707812 & 645540 & 517707 & 453868 \\
\hline ECTSP[15] & 1755886 & $(1164023)$ & 919354 & 707812 & 585771 & 517707 & 453868 \\
\hline ECTSP1[15] & 1807200 & 1228766 & 967274 & 890768 & 631115 & 562376 & 498763 \\
\hline TB-serial [6] & 1791638 & 1185434 & 912233 & 718005 & 601450 & 528925 & 455738 \\
\hline TR-serial [6] & 1853402 & 1240305 & 940745 & 786608 & 628977 & 530059 & 461128 \\
\hline TR-parallel [6] & 1975485 & 1264236 & 962856 & 800513 & 646610 & 540693 & 477648 \\
\hline K-tuple [16] & 2404341 & 1598829 & 1179795 & 1060369 & 717602 & 625506 & 491496 \\
\hline Our approach & 1752336 & 1174252 & 877977 & 703219 & 592214 & 511925 & 442478 \\
\hline
\end{tabular}

Table 3. Test time comparison on P93791.

excluded and placed within parentheses. In six out of the seven TAM bandwidths our approach finds the solution with the lowest test time. In the other case, our approach is the second best. It should be noted that the lower bound of the test time is computed assuming that the last stimuli from the previous core can be shifted out on wires concurrently as the first vector of the following core is loaded on the same wires [6]. We have in our approach not considered such optimization. Hence, our approach can be further optimized.

We have in Table 4 collected the overhead due to the use of our reconfigurable wrapper. The overhead is computed as follows. For cores with a single TAM bandwidth assigned to it, only one bandwidth is required and the cost is assumed to be zero. In some cases, only a multiplexer is to be added for the selection between wires and we assumed such cost to be equal to 1 . For cores with three configurations, we assumed the cost to be equal to 3 . We have collected the overhead at each scanned core and for the rest of the cores. The test times for the cores without scan-chains are in general shorter and in only a few cases additional logic is required.

We have also made experiments considering test power consumption. First, we illustrate the use of the RPC wrapper at core 12 assuming a fixed test time at a single TAM wire (Table 5). The test time remains the same while the test power consumption can be adjusted depending on the number of gated wrapper-chains. The added wrapper logic depends on the number of wrapper chains which indicate how many partitions are to be gated. An advantage with our scheduling approach is that we assign as few TAM wires as possible to each core, which makes it possible to have a high ratio between the number of gated wrapper chains and the number of TAM wires at each core. In other words, we have a high possibility to regulate the test power at each core.

We have compared our approach with the multiplexing and the distribution architecture [1]. In the multiplexing approach all cores are tested in a sequence where the full bandwidth is given to each core at a time. In the distribution architecture, every core is given its dedicated TAM wires. The distribution architecture is sensitive to test power consumption since the testing of all cores are started at the same time. All results are collected in Table 6. The distribution architect is not applicable when the TAM bandwidth is below the number of cores (32 in P93791). At the 50000 power limit the distribution architecture can not be used since activating all cores exceeds the power limit. At the limit 20000, the multiplexing approach is not applicable since core 6 limits the solution with its consumption of 24674. Our approach results in the same test time, however, the wrapper logic is increased in order to gate the wrapper chains. 


\begin{tabular}{|c|c|c|c|c|c|c|c|c|c|c|c|c|c|c|c|}
\hline \multirow{2}{*}{$\begin{array}{c}\text { TAM } \\
\text { band-width }\end{array}$} & \multirow[b]{2}{*}{ Test time } & \multicolumn{14}{|c|}{ Wrapper logic at core $c_{i}$} \\
\hline & & $\mathbf{c}_{1}$ & $\mathbf{c}_{6}$ & $\mathbf{c}_{11}$ & $\mathbf{c}_{12}$ & $\mathbf{c}_{13}$ & $\mathbf{c}_{14}$ & $\mathbf{c}_{17}$ & $\mathbf{c}_{19}$ & $\mathbf{c}_{20}$ & $\mathbf{c}_{23}$ & $\mathbf{c}_{27}$ & $c_{29}$ & $\begin{array}{c}\text { Cores with } \\
\text { no scan chains }\end{array}$ & Total \\
\hline 4 & 6997584 & 0 & 1 & 0 & 0 & 0 & 1 & 0 & 0 & 1 & 0 & 0 & 0 & 0 & 3 \\
\hline 8 & 3498611 & 0 & 3 & 0 & 1 & 0 & 1 & 0 & 1 & 1 & 0 & 1 & 0 & 1 & 9 \\
\hline 16 & 1752336 & 1 & 3 & 0 & 1 & 1 & 1 & 1 & 1 & 3 & 1 & 1 & 1 & 1 & 16 \\
\hline 24 & 1174252 & 2 & 3 & 0 & 3 & 3 & 3 & 3 & 1 & 3 & 3 & 3 & 1 & 1 & 29 \\
\hline 32 & 877977 & 2 & 3 & 1 & 3 & 3 & 3 & 3 & 3 & 3 & 3 & 3 & 3 & $1+1$ & 35 \\
\hline 40 & 703219 & 2 & 3 & 0 & 3 & 3 & 3 & 3 & 3 & 3 & 3 & 3 & 3 & $3+1$ & 36 \\
\hline 48 & 592214 & 2 & 3 & 0 & 3 & 3 & 3 & 3 & 3 & 3 & 3 & 3 & 3 & $3+2$ & 37 \\
\hline 56 & 511925 & 2 & 3 & 0 & 3 & 3 & 3 & 3 & 3 & 3 & 3 & 3 & 3 & $3+2+3$ & 40 \\
\hline 64 & 442478 & 2 & 3 & 1 & 3 & 3 & 3 & 3 & 3 & 3 & 3 & 3 & 3 & $3+3+3$ & 42 \\
\hline
\end{tabular}

Table 4. Number of configurations per core for our scheduling approach on P93791 (Table 3).

\section{Conclusions}

In this paper we have proposed a reconfigurable powerconscious core test wrapper, and described its application to preemptive test scheduling. The main advantages with the wrapper is the possibility to (1) control the test power consumption at each individual core and (2) the possibility to achieve several TAM bandwidths for a given core test. Test power control at each core is important since it allows testing at a higher clock frequency, which can be used to further decrease the test time. Test power control also allows a higher number of cores to be tested simultaneously. Flexible bandwidth is important since it increases the flexibility during the scheduling process. The advantage of the proposed test scheduling scheme, besides the production of an optimal solution in respect to test time, is that it also considers cross-core testing, which are used to test unwrapped testable units such as interconnections and used-defined logic.

\begin{tabular}{ccc}
\hline Wrapper chains & Power consumption & Wrapper logic \\
\hline 1 & 4634 & 0 \\
2 & 2317 & 1 \\
3 & 1545 & 3 \\
4 & 1159 & 4 \\
5 & 927 & 5 \\
6 & 773 & 6
\end{tabular}

Table 5. Test power consumption options at core 12 (P93791) with the RPC wrapper at a fixed test time $(=1813502)$ and fixed TAM bandwidth (=1).

\section{References}

[1] J. Aerts and E. J. Marinissen, "Scan Chain Design for Test Time Reduction in Core-Based ICs", Proceedings of International Test Conference (ITC), pp. 448-457, October 1998.

[2] P. Brucker, "Scheduling Algorithms", Springer-Verlag, ISBN 3-540-64105-X, 1998.

[3] M. L. Bushnell and V. D. Agrawal, "Essentials of Electronic Testing for Digital, Memory, and Mixed-Signal VLSI Circuits", Kluwer Academic Publisher, ISBN 0-7923-79918.

[4] R. Chou, K. Saluja, and V. Agrawal, "Scheduling Tests for VLSI Systems under Power Constraints", Transactions on VLSI Systems, Vol. 5, No. 2, pp. 175-185, June 1997.

[5] S. Gerstendörfer and H-J Wunderlich, "Minimized Power Consumption for Scan-Based BIST", Proceedings of International Test Conference (ITC), pp. 77-84, Atlantic City, NJ, September 1999.

[6] S. K. Goel and E. J. Marinissen, "Effective and Efficient Test Architecture Design for SOCs", Proceedings of International Test Conference (ITC), pp 529-538, October 2002.

[7] S. K. Goel and E.J. Marinissen, "A Novel Test Time Reduction Algorithm for Test Architecture Design for coreBased System Chips", Digest of papers European Test Workshop (ETW), pp 41-46, Corfu, Greece, May 2002.

[8] S. K. Goel and E. J. Marinissen, "Cluster-Based Test Architecture Design for System-On-Chip, Proceedings of VLSI Test Symposium (VTS), pp. 259-264, Monterey, California, April 2002.

[9] Y. Huang, S. M. Reddy, W.-T. Cheng, P. Reuter, N. Mukherjee, C.-C. Tsai, O. Samman, and Y. Zaidan, "Optimal Core Wrapper Width Selection and SOC Test Scheduling Based on 3-D Bin Packing Algorithm", Proceedings of International Test Conference (ITC), pp. 74-82, Baltimore, Maryland, USA, October 2002. 
[10] Y. Huang, W.-T. Cheng, C.-C. Tsai, N. Mukherjee, O. Samman, Y. Zaidan and S. M. Reddy, "Resource Allocation and Test Scheduling for Concurrent Test of Core-based SOC Design", Proceedings of IEEE Asian Test Symposium (ATS), pp 265-270, Kyoto, Japan, November 2001.

[11] V. Iyengar, K. Chakrabarty, and E. J. Marinissen, "Wrapper/ TAM Co-Optimization, Constraint-Driven Test Scheduling, and Test Data Volume Reduction for SOCs", Proceedings of IEEE/ACM Design Automation Conference (DAC), pp. 685690, New Orleans, Louisiana, June 2002.

[12] V. Iyengar, K. Chakrabarty, and E. J. Marinissen, "CoOptimization of Test Wrapper and Test Access Architecture for Embedded Cores", Journal of Electronic Testing; Theory and Applications (JETTA), pp 213-230, April 2002.

[13] V. Iyengar K. Chakrabarty, and E. J. Marinissen, "Efficient Wrapper/TAM Co-Optimization for Large SOCs", Proceedings of Design and Test in Europe (DATE), pp. 491498, Paris, France, March 2002.

[14] V. Iyengar, K. Chakrabarty, and E. J. Marinissen, “On Using Rectangle Packing for SOC Wrapper/TAM CoOptimization", Proceedings of VLSI Test Symposium (VTS), pp. 253-258, Monterey, California, April 2002.

[15] S. Koranne, "A Novel Reconfigurable Wrapper for Testing Embedded Core-Based and its Associated Scheduling", Journal of Electronic Testing; Theory and Applications (JETTA), pp. 415-434, August 2002.

[16] S. Koranne and V. Iyengar, "On the Use of k-tuples for SoC Test Schedule Representation", Proceedings of International Test Conference (ITC), pp. 539-548, October 2002.

[17] E. Larsson and H. Fujiwara, "Optimal Test Access Mechanism Scheduling using Preemption and Reconfigurable Wrappers", Proceedings of IEEE Workshop on RTL and High Level Testing (WRTLT), Guam, USA, November 21-22, 2002.

[18] E. Larsson and Z. Peng, "An Integrated Framework for the Design and Optimization of SOC Test Solutions", Journal of Electronic Testing; Theory and Applications (JETTA), pp. 385-400, August 2002.

[19] E. J. Marinissen, R. Kapur, M. Lousberg, T. McLaurin, M. Ricchetti, and Y. Zorian, “On IEEE P1500's Standard for Embedded Core Test", Journal of Electronic Testing: Theory and Applications, (JETTA), vol. 18, pp 365-383, August 2002.

[20] E. J. Marinissen, V. Iyengar, and K. Chakrabarty, "ITC'02 SOC Test Benchmarks Web Site", http:// www.extra.philips.com/itc02socbench/.

[21] N. Nicolici and B. M. Al-Hashimi, "Power Conscious Test Synthesis and Scheduling for BIST RTL Data Paths", Proceedings of International Test Conference (ITC), pp. 662671, Atlantic City, NJ, Oct. 2000.

[22] P. M. Rosinger, B. M. Al-Hashimi, and N. Nicolici, "Scan Architecture for Shift and Capture Cycle Power Reduction", Proceedings of IEEE International Symposium on Defect and Fault Tolerance in VLSI Systems, pp. 129-137, 2002.
[23] P. M. Rosinger, B. M. Al-Hashimi, and N. Nicolici, "Power Profile Manipulation: A New Approach for Reducing Test Application Time under Power Constraints", Transactions on Computer-Aided Design of Integrated Circuits and Systems, pp. 1217-1225, October 2002.

[24] J. Saxena, K. M. Butler, and L. Whetsel, "An Analysis of Power Reduction Techniques in Scan Testing", Proceedings of International Test Conference (ITC), pp. 670-677, Baltimore, MD, October 2001.

\begin{tabular}{|c|c|c|c|c|}
\hline \multirow{2}{*}{$\mathbf{P}_{\max }$} & \multirow{2}{*}{ TAM } & $\begin{array}{l}\text { Multiplexing } \\
\text { architecture }\end{array}$ & $\begin{array}{l}\text { Distribution } \\
\text { architecture }\end{array}$ & Our approach \\
\hline & & Test time & Test time & Test time \\
\hline \multirow{9}{*}{100000} & 4 & 7113317 & Not applicable & 6997584 \\
\hline & 8 & 3625510 & Not applicable & 3498611 \\
\hline & 16 & 1862427 & Not applicable & 1752336 \\
\hline & 24 & 1262427 & Not applicable & 1174252 \\
\hline & 32 & 1210398 & 5317007 & 877977 \\
\hline & 40 & 1119393 & 1813502 & 703219 \\
\hline & 48 & 660143 & 1126316 & 592214 \\
\hline & 56 & 645698 & 907097 & 511925 \\
\hline & 64 & 645682 & 639989 & 442478 \\
\hline \multirow{9}{*}{50000} & 4 & 7113317 & Not applicable & 6997584 \\
\hline & 8 & 3625510 & Not applicable & 3498611 \\
\hline & 16 & 1862427 & Not applicable & 1752336 \\
\hline & 24 & 1262427 & Not applicable & 1174252 \\
\hline & 32 & 1210398 & Not applicable & 877977 \\
\hline & 40 & 1119393 & Not applicable & 703219 \\
\hline & 48 & 660143 & Not applicable & 592214 \\
\hline & 56 & 645698 & Not applicable & 511925 \\
\hline & 64 & 645682 & Not applicable & 442478 \\
\hline \multirow{9}{*}{20000} & 4 & Not applicable & Not applicable & 6997584 \\
\hline & 8 & Not applicable & Not applicable & 3498611 \\
\hline & 16 & Not applicable & Not applicable & 1752336 \\
\hline & 24 & Not applicable & Not applicable & 1174252 \\
\hline & 32 & Not applicable & Not applicable & 877977 \\
\hline & 40 & Not applicable & Not applicable & 703219 \\
\hline & 48 & Not applicable & Not applicable & 592214 \\
\hline & 56 & Not applicable & Not applicable & 511925 \\
\hline & 64 & Not applicable & Not applicable & 442478 \\
\hline
\end{tabular}

Table 6. Test time on P93791 for the multiplexing architecture [1], the distribution architecture [1], and our approach at different power limitations. 